\title{
Simulation of the Effect of a Series of Superconducting Magnets on a Quenching Magnet Using a Controlled Current Pulse
}

\author{
A. Hilaire, A. Ijspeert, and J. C. Perez
}

\begin{abstract}
In the LHC, the superconducting corrector magnets will be powered in series of up to 154 magnets. For protection in case of a quench, each magnet has been equipped with a parallel resistor as a bypass for the current. To validate and optimize the parallel resistor value, a test arrangement has been set up which allows quenching a single magnet as if it were connected in a large series of magnets. This simulation is obtained by maintaining the current for a certain time interval after the quench occurred. Calculations have shown that, depending on the magnet type, a current duration (after quench) of $0.2 \mathrm{~s}$ to $1 \mathrm{~s}$ simulates correctly the effect of the series of magnets. The paper gives calculation results comparing the real situation with the simulated one and reports on the test set-up that will be used to optimize the parallel resistors.
\end{abstract}

\section{INTRODUCTION}

M ORE than 6000 superconducting corrector magnets are used in the LHC and will be powered in around 1500 electrical circuits. A number of these circuits feed individual magnets but in some, the magnets will be powered in series of up to 154 units. In case of a quench, the corrector magnets are designed to absorb their own energy without damage. However when they are connected in series, an additional protection has to be foreseen. It consists of a parallel resistor that bypasses the current flowing from the nonquenching magnets of the series. In addition, an external resistance is switched into the circuit to extract energy and hence reduce the energy deposition in the quenching magnet and its associated resistor.

Quench calculations have been done [1] and experiments were performed on single magnets. However, to be able to simulate the effect of a chain of magnets, it has been decided to make a new test set-up. It uses a strong power supply that can maintain the current when the magnet quenches even though the voltage may rise to $150 \mathrm{~V}$. This allows to maintain the current over a time such that the extra energy produced by the power supply corresponds to the energy of the series of magnets. The individual magnet with its parallel resistor is quenched by means of a spot heater.

Calculations have been made to compare the real situation in the machine with the test situation and the results are reported and the test set-up is described.

Manuscript received September 24, 2001.

The authors are with CERN, European Organization for Nuclear Research, 1211 Geneva 23, Switzerland.

Publisher Item Identifier S 1051-8223(02)04236-7.

\section{TYPES OF MAGNET}

The magnets concerned are the Tuning Quadrupoles (MQT, MQS), the arc Chromaticity Sextupoles (MS, MSS), the Spool Correctors (MCS, MCO, MCD) that correct the fields of the main bending magnets, the Landau damping Octupoles (MO) and the Long Trim Quadrupoles (MQTL). All these magnets with the exception of the MQTL can be tested in our cryostat. The MQTL being a long version of the MQT, the results of the latter will be extrapolated to the MQTL. MQS and MSS are tilted versions of MQT and MS magnets respectively.

On each magnet type, computer calculations have been made for the different circuits used in the LHC $(1,2,4$, and 8 MQT in series, 1, 2 and $6 \mathrm{MQTL}, 4$ and $12 \mathrm{MS}, 13 \mathrm{MO}, 154 \mathrm{MCS}$ and $77 \mathrm{MCO}$ or MCD).

\section{QUENCH CALCULATIONS}

\section{A. Computer Simulations}

Quench calculations have been made using the CERN-developed program QUABER [2] to model the quench distribution in a magnet and to find the current decay as a function of the time.

Electrically, a variable resistance and an inductance in series simulate each coil of the quenching magnet. A quench propagates from one coil to the next by thermal conduction. This thermal conduction is longitudinal (along the length of the superconducting wire) or transversal, across the insulation layer. The quench starts in one of the coils and is transmitted to the neighboring coils with delays depending on the superconducting wire used and on the number of turns per coil.

Raw data obtained (temperature, voltages, currents, and voltages across the resistive part of the quenching coil) were then analyzed to find the evolution of the magnet resistance and of the Miits dissipated in the quenching magnet. The current and energy dissipated in the parallel resistor have also been computed.

\section{B. Three Cases of Simulation}

For each type of corrector magnet, three cases have been calculated.

The first case corresponds to the circuit in the accelerator (see Fig. 1). The power supply is switched off at the start of the quench and the current in the quenching magnet decreases according to the time constant of the circuit (magnet inductances and parallel resistors). If needed the energy extraction resistor is switched into the circuit. 
TABLE I

MAIN PARAMETERS OF THE MAGNETS

\begin{tabular}{ccccccc}
\hline Name & Type & $\begin{array}{c}\text { Family } \\
\text { Size }\end{array}$ & $\begin{array}{c}\text { Current } \\
\text { (A) }\end{array}$ & $\begin{array}{c}\text { Induct } \\
\text { (H) }\end{array}$ & $\begin{array}{c}\text { R para } \\
\text { (o) }\end{array}$ & Extr. \\
\hline MQTL & Quad. & $1 \ldots 6$ & 550 & 120 & 0.2 & Yes \\
MQT/MQS & Quad. & $2 \ldots 8$ & 550 & 31 & 0.25 & Yes \\
MS IMSS & Sext. & $4 \ldots 12$ & 550 & 36 & 0.15 & Yes \\
MO & Oct. & $8 \ldots 13$ & 550 & 1.5 & Ho & Yes \\
MCS & Sext. & 154 & 550 & 0.8 & 0.08 & Yes \\
MCD & Deca. & 77 & 550 & 0.4 & No & Yes \\
MCO & Oct. & 77 & 100 & 0.4 & No & No \\
\hline
\end{tabular}

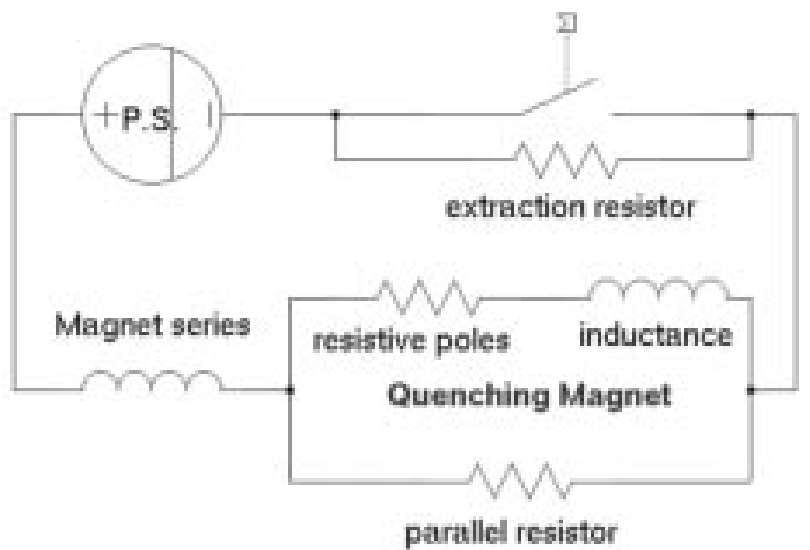

Fig. 1. Schematic circuit used for the simulations.

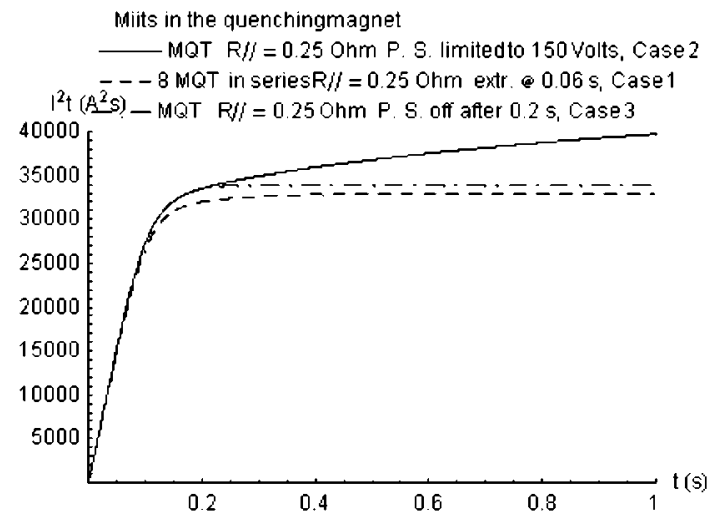

Fig. 2. Miits calculated for the quench of an MQT tuning quadrupole.

The second case (same as Fig. 1 but the "magnet series" and "extraction resistor" are suppressed) does not incorporate the rest of the family but uses a constant current source that is kept on permanently. This calculation enables to verify the value of the parallel resistor to be sufficiently low to assure that after a certain time all the current by-passes the magnet.

The third case corresponds to the validation simulating test. It is like the previous case but the current source is switched off at the moment that the Miits in the magnet correspond to the value found in the first, more realistic case.

An example of such simulations is given in Fig. 2. The simulation is done for MQT Tuning Quadrupole magnets. The curves represent the Miits in the quenching magnet as a function of time; the top curve is the result for case 2 where the powersupply is kept on, below follow cases 1 and 3, respectively. It can be seen that the parallel resistor is efficient because the Miits

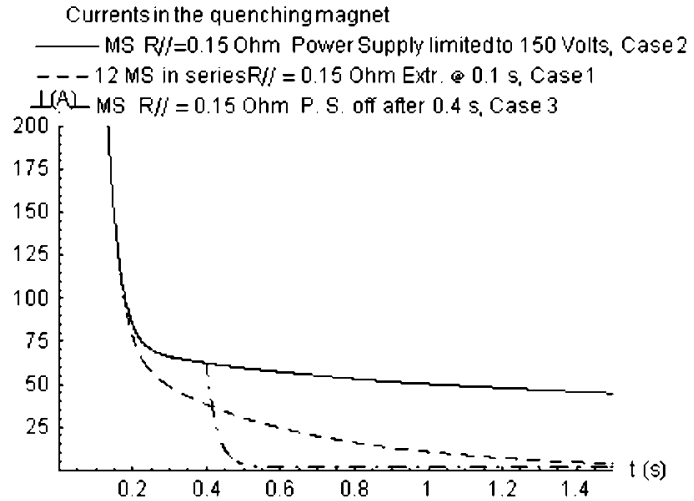

Fig. 3. Current inside the quenching MS sextupole.

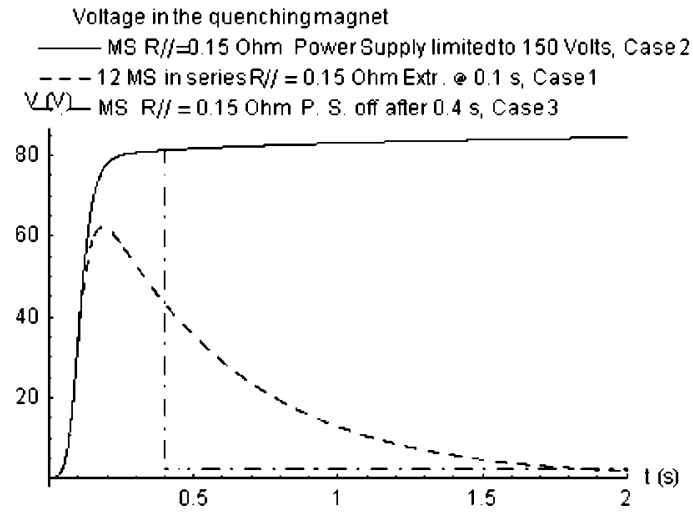

Fig. 4. Voltage across the MS sextupole.

do not increase much after the magnet has completely quenched (after $\sim 0.3 \mathrm{~s}$ ).

The temperature increase and the voltages must stay below certain levels considered to be safe. The voltage allowance is 200 Volts between coils and the allowed peak temperature is $220-230 \mathrm{~K}$. These limits have been determined by experiment.

\section{CAlculated Results}

The main objective of these calculations was to find delays of the power supply that correspond to the effect of a family of magnets.

Using the Quaber results, one can obtain the voltage across the magnet, the resistive voltages, the resistance of the quenching magnet and the current, all functions of time. Some examples of such analyses are given below.

The current inside the quenching MS magnet is shown in Fig. 3. The current decay for the 3 cases is the same in the first period of some $0.2 \mathrm{~s}$. At the end of this period, the current in the magnet is only one eighth of the starting value of $600 \mathrm{~A}$ and the quench of the magnet is almost complete. In the case where the power-supply stays on (case 2), still one tenth of the current continues to pass permanently through the quenching magnet. In cases 1 and 3, one sees that, once the power-supply is switched off, the current driven by the power supply decreases faster than that of the "natural quench" in the LHC due to the different time constants of the circuits (one versus 12 magnets). 


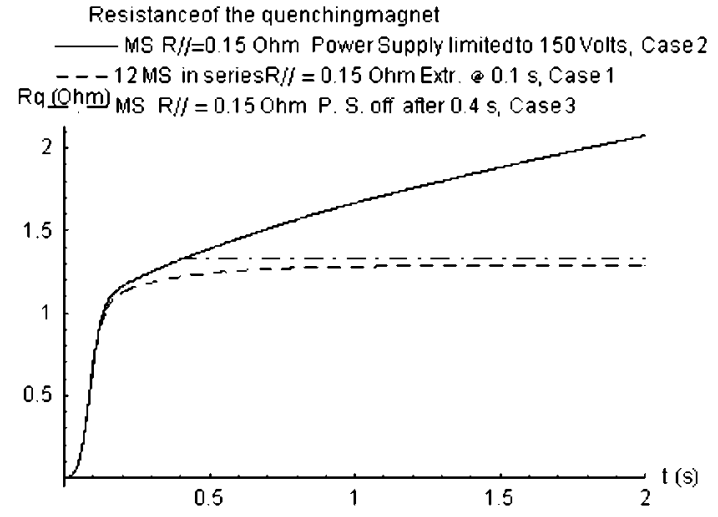

Fig. 5. Resistance of the MS sextupole.

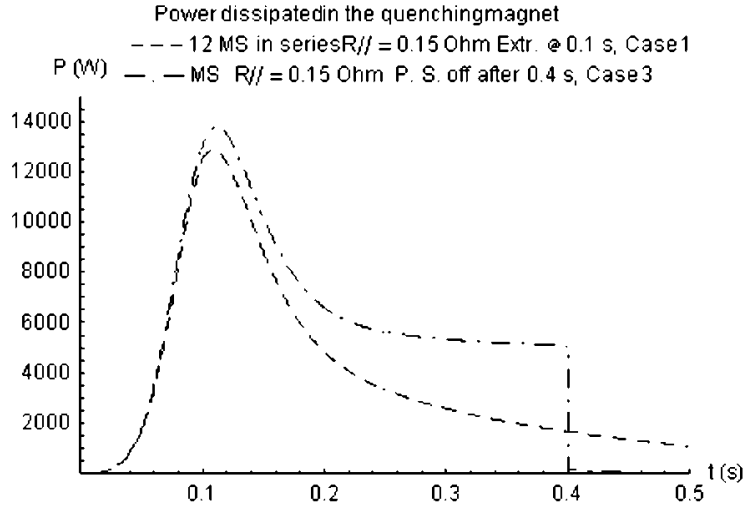

Fig. 6. Power dissipated in the MS sextupole.

The voltage across the quenching magnet is shown in Fig. 4 . The "natural quench" gives a lower voltage but with a longer duration. The duration is due to the chain of magnets which deposits its energy in the quenching one. The power supply, in the case of the test, imposes a higher voltage on the magnet. Once the power supply is switched off however, the voltage is determined by the equivalent resistance of the switched off power supply and practically zero.

The resistance in the quenching magnet is shown in Fig. 5. Due to the faster decay of the current in the "natural quench," during the first period (Fig. 3), the resistance in the magnet saturates faster. If the power supply continues to feed the magnet (case 2), the resistance continues to increase whereas when the power supply is switched off (case 3), the resistance does not vary anymore as the small part of the current passing through the magnet vanishes.

The power dissipated in the quenching magnet is shown in Fig. 6. Only the "natural quench" and the one induced by the power supply cut after $0.4 \mathrm{~s}$ are shown. One can see that during the main part of the quench, the power dissipations are comparable.

As shown in these examples one can simulate the behavior of a series of magnets sufficiently well by keeping the current of the power-supply on for a given time interval. The delay for switching off the power supply has to be adjustable and the precision for this delay has to be of the order of a millisecond.
TABLE II

Calculated Mits and MaXimum Temperatures IN the QUENCHING MAGNETS

\begin{tabular}{|c|c|c|c|c|c|}
\hline & \multicolumn{2}{|c|}{$\begin{array}{c}\text { Case } 1 \\
\text { Natural quench }\end{array}$} & \multicolumn{3}{|c|}{$\begin{array}{c}\text { Case } 3 \\
\text { Simulation with port er supp dy Delay }\end{array}$} \\
\hline & Temp $(\mathrm{K})$ & Mits & Delay (s) & $\begin{array}{l}\text { Temp. (K) } \\
\text { With P.S. }\end{array}$ & $\begin{array}{l}\text { Mints } \\
\text { with P.S }\end{array}$ \\
\hline $\mathrm{MQTL}$ & 227 & 33500 & & & \\
\hline & 219 & 32900 & 0.2 & 232 & 33800 \\
\hline M SiM SS & 222 & 32700 & 0.4 & 241 & 36000 \\
\hline $\mathrm{M} 0$ & 130 & 25500 & 0.07 & 156 & 28400 \\
\hline MCS & 176 & 30900 & 0.15 & 198 & 32800 \\
\hline$M C D$ & 159 & 29100 & 0.07 & 155 & 28700 \\
\hline$M C 0$ & 211 & 4200 & 0.29 & 225 & 4320 \\
\hline
\end{tabular}

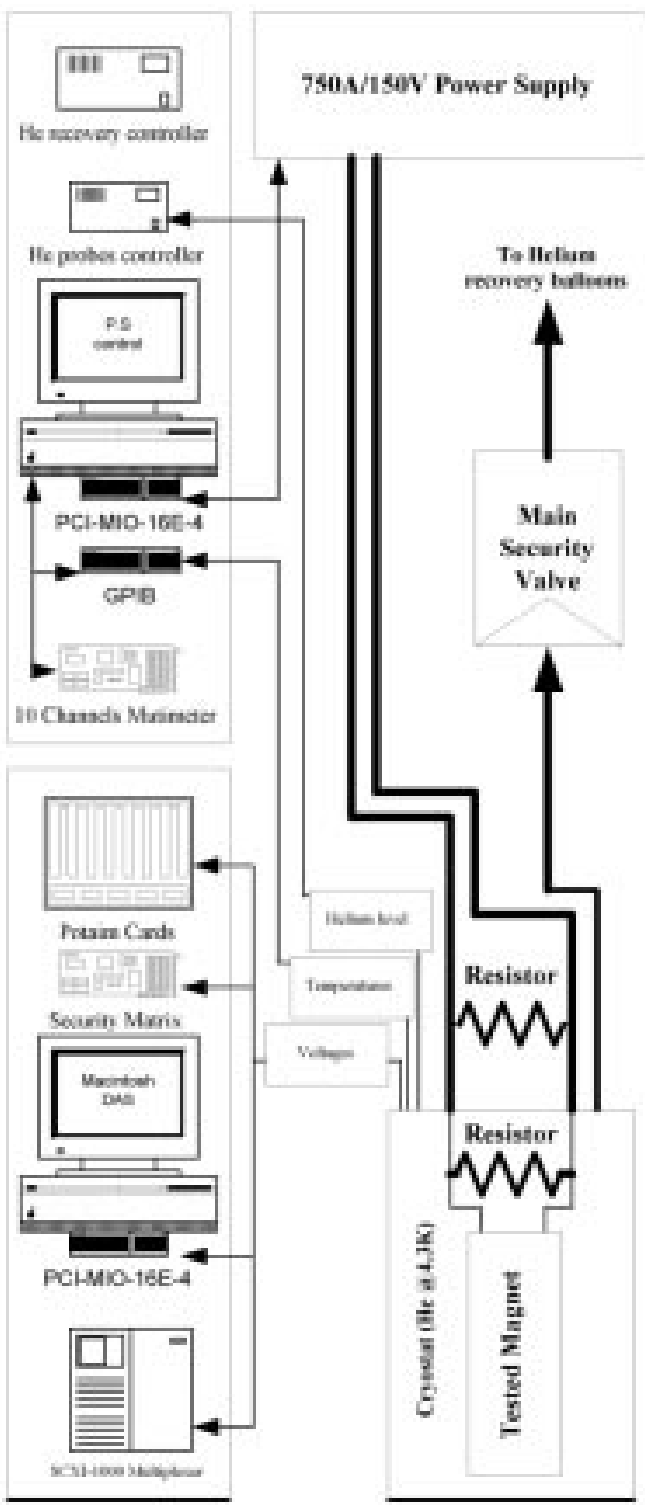

Fig. 7. The experimental set-up.

\section{RESUlts SUMMARIZED}

Table II shows a summary of the Miits and maximum temperatures reached in the different types of corrector magnets.

The first two columns correspond to the calculation of the chain of magnets in the ring (case 1 the "natural quench"). The other ones give the delay to switch off the power supply (third column) and the Miits and temperature reached in the magnets under these conditions (case 3). 


\section{THE EXPERIMENTS}

A dedicated cryogenic test station has been built to verify that the protection resistors effectively avoid any risk of damage to the magnets in case of a quench. The tests must give the evidence that the value of the protection resistor is low enough to allow the current to by-pass the resistive magnet, without being too low to avoid important leak currents during ramping up of the field. The magnet on test, equipped with its parallel resistor, is mounted in a cryostat. For safety the test is started with an additional second parallel resistor of the same value mounted outside the cryostat between the warm connections. In this way we start the test with an increased protection (total by-pass resistance halved) and this second resistor is taken away once the tests perform well. The magnet is powered first at a current below nominal, a quench is induced firing the spot heater and the power converter is stopped a few milliseconds later. This test is repeated, increasing the time of power-on after quench, and the Miits in the magnet are recorded as an indication for the peak temperature in the magnet (as it is extremely difficult to measure this temperature directly). This scenario is repeated at higher currents and finally at nominal current. The Miits must stay acceptable, as is the case for the magnet absorbing its own energy, and should not increase significantly as a function of the time of power-on.

\section{EXPERIMENTAL SET-UP}

The experimental set up is shown schematically in Fig. 7.

An existing cryostat has been adapted to perform the different tests. The current flowing in the magnet has to be maintained after the quench for times up to $400 \mathrm{~ms}$. In certain cases, when the tested magnet becomes resistive, the dissipated energy can evaporate up to 501 of liquid helium, giving approximately $35 \mathrm{~m}^{3}$ of helium gas. This gas needs to be extracted in order to prevent excessive pressure build-up in the cryostat. A special mechanical safety valve has been developed. In case of over-pressure in the cryostat, the gas pressure opens the valve and fills up to two recovery balloons of $40 \mathrm{~m}^{3}$ each. In case of failure of this system, two secondary safety valves will open should the internal pressure exceed 1.7 bars absolute.

Two vapor cooled $1500 \mathrm{~A}$ current leads connect the magnet under test to a $150 \mathrm{~V} / 700$ A power supply.

To generate a quench, two small flat resistors are glued on the coil ends of the magnet. By discharging a capacitor, these resistances act as spot heaters and generate enough energy to produce a local resistive transition in the superconducting coil.

A data acquisition program has been developed to monitor voltage and current and store the data once triggered by the quench. From the moment of quench, the switching off of the power supply can be postponed from 10 to $1000 \mathrm{~ms}$ to simulate the discharge of a magnet family as in the final layout of the LHC machine.

\section{CONCLUSION}

It has been shown that the protection of corrector magnets connected in series can be tested on a single magnet simulating the effect of the inductance of the family through a power supply that can maintain the current during the quench. An experimental set-up has been made and the test program is presently under way.

\section{ACKNOWLEDGMENT}

The authors wish to thank A. Rijlaert, C. Charrondière, and F. Vachez for the preparation of the necessary instrumentation and control programs.

\section{REFERENCES}

[1] R. Schmidt et al., "Protection of the superconducting corrector magnets for the LHC," in EPAC 2000, Vienna, Austria.

[2] D. Hagedorn and F. Rodriguez-Matteos, "Modeling of the quenching process in complex sc magnet systems," IEEE Trans. Magn., vol. 28, Jan. 1992. 